Al-Azhar Bull. Sci. Vol. 18, No. 2 (Dec.): pp. 95-104, 2007.

\title{
NEW EFFECTS OF PRINTING COTTON/POLYESTER BLENDED FABRICS USING ALKALI PRINTING
}

\author{
Y.H.ELHAMAKY ${ }^{(1)}$ S.H.NASSAR ${ }^{(2)}$ H.A.OSMAN ${ }^{(3)}$ D.F.IBRAHIM ${ }^{(1)}$ \\ 1. Faculty of Applied Arts, Helwan University \\ 2. National Research Centre,Textile Research Division, Dokki, Cairo, Egypt \\ 3. Faculty of Specific Education,Banha,Egypt
}

\begin{abstract}
New printing effects on cotton/polyester blended fabrics were achieved by printing the fabrics using a printing paste containing different concentrations of sodium hydroxide, and then steamed applying different steaming time and temperatures, in order to improve the two fabrics physical properties. These changes resemble in: Decreasing fabric weight, increasing water absorbency and air permeability, shrinkage effect which cause the differential crimping effect and color tones. Factors affecting these new effects were studied and discussed in details to determine the most desirable appearance of cotton / polyester blended fabric.
\end{abstract}

\section{Introduction:}

Cotton / polyester blended fabrics have taken a major position in the field of blended fabrics. In an attempt to improve the consumer acceptance of polyester fabrics, cotton yarns were blended with polyester to improve comfort and better dye ability while maintaining the wrinkle resistance and dimensional stability of 100 percent polyester. ${ }^{(1)}$ At the same time, some technical difficulties arose in blending process such as; some lake of uniformity in yarn, which may be ascribed to differences in specific gravity, fiber length, diameter, surface shape, texture and hygroscopicity of the fiber components. Commonly, the most popular ratio of polyester blended with cotton fibers are 65 / 35 and 50 / 50 respectively. ${ }^{(2)}$

Alkali treatments of $100 \%$ polyester fabrics were used in many trails to improve polyester properties; which may be assessed in terms of substrate weight loss, improving of handle and wetabilitty properties. ${ }^{(3)} 100 \%$ Cotton fabrics were also alkali treated (mercerization) altering luster, moisture absorbency and strength with both, shrinking and swelling effect. ${ }^{(4)}$

In previous work, cotton / polyester blended fabrics were printed with printing paste including sodium hydroxide (at different conditions) in order to improve both fibers (cotton and polyester) properties. Then the blended fabric was dyed with disperse dye as a way to achieve different colour tones which gives, in addition to the crimping effect, the new fashion look. Some effective factors and measurements were studied and discussed in details. 


\section{Experimental work:}

\section{Materials and chemicals:}

\section{Fabrics}

Polyester / cotton union cloth 50/50 \%, supplied by El -shorpagy for spinning and weaving company, Egypt. was used through out this work. Using 150/1 (Denier) warps bleached polyester yarns and count 20/1 (En) for weft (combed cotton yarns). Weaving structure applied is plain weave $1 \backslash 1$. Weight of square meter is $108 \mathrm{~g} / \mathrm{m}^{2}$.

Dyestuffs:

One disperse dye namely;

- Foron yellow RD4 GLS GR supplied by Zeneca, England, was used.

\section{Chemicals:}

Sodium hydroxide, acetic acid, dispersing agent and carrier from Morgan Chemicals Co. Egypt.

Thickening agents:

Meypro - Gum NP-16 from Meyhall chemical AG CH - 8280 Kreuzllngen । Switzerland.

\section{Procedures:}

The blended cotton/polyester fabrics were printed with printing paste thickened with meypro-gum in presence of sodium hydroxide by flat screen (stripes). After drying, steaming in laboratory steamer, blended fabrics were washed and soaped, then dried and finally were dyed with disperse dye in the following dyeing bath contents:

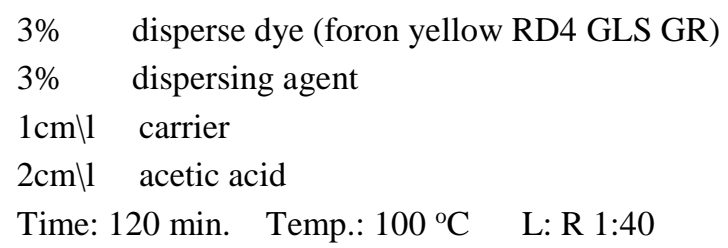

After dyeing, the blended cotton/polyester fabrics were washed then soaped in soaping bath contains $1 \mathrm{~g} / \mathrm{l}$ nonionic detergent (Hospatal) for $15 \mathrm{~min}$ at boiling, and then it were rinsed and dried. 


\section{Measurements:}

- $\quad$ Measurement of weight loss :

The weight loss of the sample was calculated by the formula:

$$
\text { Weight loss }(\%)=\frac{\mathrm{W}_{1}-\mathrm{W}_{2}}{\mathrm{~W}_{1}} * 100
$$

Where,

$\mathrm{W}_{1}=$ weight before treatment, and

$\mathrm{W}_{2}=$ weight after treatment.

- Water repellency of the samples was determined on a spray tester (Water Repellency_tester)__ model no. BS370 2I S04920.

- $\quad$ Fabric air permeability Tester model. ASTMD 737 was used.

- $\quad$ Fabric drape meter determined on a Cusick Drape tester AS.T.M standards D4032 - 75 .

- $\quad$ The colour strength $(K \backslash S)$ of the dyed samples is assessed according to the standard technique.

\section{Result and discussion:}

\section{Effect of NaOH concentrations:}

Different concentrations of $\mathrm{NaOH}$ were added to the printing paste used in printing cotton / polyester blended fabrics to determine the most suitable concentration of alkali which has the desirable effect on some of both fabric properties, such as loss in weight, tensile strength, fabric drapeability, air permeability, water repellency, and fabric shrinkage. Table (1) and fig.(1-A and 1-B) show the result of using different concentrations of $\mathrm{NaOH}$ in printing paste of the blended cotton / polyester fabric, which is steamed at $115{ }^{\circ} \mathrm{C}$ for 20 minutes. 
Table (1) Effect of $\mathrm{NaOH}$ conc. on the properties of printed cotton/polyester blended fabrics

\begin{tabular}{|c|c|c|c|c|c|c|}
\hline \multirow{2}{*}{$\begin{array}{c}\mathrm{NaOH} \\
\text { conc.(g/kg) }\end{array}$} & \multirow{K}{*}{$\mathrm{K} \backslash \mathrm{S}$} & $\begin{array}{c}\text { Loss in } \\
\text { weight } \\
\end{array}$ & & \multirow{2}{*}{$\begin{array}{c}\text { Drape } \\
\text { meter }\end{array}$} & \multirow{2}{*}{$\begin{array}{c}\text { Air permeability } \\
\left(\mathrm{cm}^{3} / \mathrm{cm}^{2} / \mathrm{s}\right)\end{array}$} & \multicolumn{2}{|c|}{ Water Repellency } \\
\cline { 6 - 7 } & & & & Absorption & Repellent \\
\hline With out & 0.64 & 0 & 0.63 & 63.20 & $50 \%$ & $50 \%$ \\
\hline 10 & 0.73 & 3.30 & 0.56 & 64.33 & $60 \%$ & $40 \%$ \\
\hline 15 & 0.76 & 6.78 & 0.51 & 65.52 & $70 \%$ & $30 \%$ \\
\hline 20 & 0.77 & 13.0 & 0.46 & 66.75 & $80 \%$ & $20 \%$ \\
\hline 25 & 0.84 & 20.0 & 0.42 & 67.98 & $90 \%$ & $10 \%$ \\
\hline 30 & 0.89 & 26.6 & 0.35 & 68.60 & $95 \%$ & $5 \%$ \\
\hline
\end{tabular}
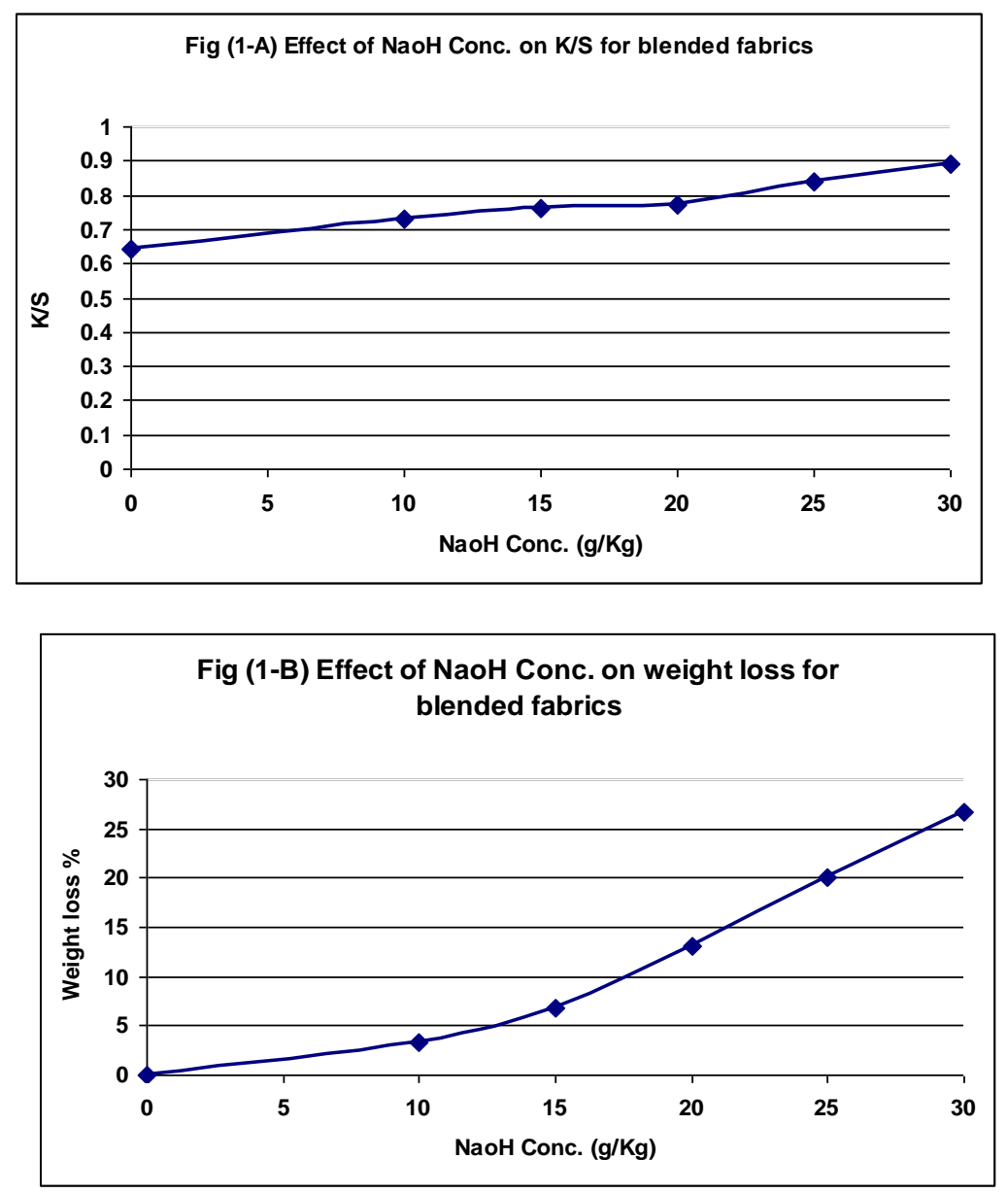
From the previous figures and table, it is found that increasing alkali concentration in the printing paste of the blended cotton/polyester fabric produces an increase in fabric weight loss, in colour strength, air permeability and water absorption of the printed areas.

Using of $(25 \mathrm{~g} / \mathrm{kg})$ sodium hydroxide in printing paste of blended fabric was found to be the most suitable concentration. At this level a suitable weight loss (20 $\%$ ) and an increasing in colour strength for the (printed, then dyed areas) reached (32 $\%$ ), as a result of wettability and dyeability properties improvement. This was due to alkali effect on the fabric surface, at which hydroxyl ions of sodium hydroxide attack the carbonyl groups of polyester fibers and decreased its hydrophobic character. As a result of weight reduction, the fiber diameter became smaller and the dyes can diffuse more easily. Any increase in alkali concentration in the printing paste caused more weight loss which decreased the blended fabric tensile strength. ${ }^{(5}$, 6)

Concerning other physical properties, it was found that decreasing in drapeability for about (33\%) may be due to the loss in weight which made the fabric lighter and less draping. Air permeability seemed to increase from $\left(63.20\right.$ to $\left.67.9 \mathrm{~cm}^{3} / \mathrm{cm}^{2} / \mathrm{s}\right)$ according to the weight loss of polyester yarns which increased the spaces between cotton and polyester yarns causing increasing in air permeability.

Water repellency of blended fabrics differed after alkali printing, as a result of removing the polyester fabric hydrophobic surface which contains carbonyl groups It was found that absorption of the fabric increased from (50\% to $90 \%)$ so repellency decreased from $(50 \%$ to $10 \%)$.

Concerning alkali effect on cotton fibers in these blended fabrics, the next photos could clearly show the difference between the blended fabrics which were printed with $(10 \mathrm{~g} / \mathrm{kg}$ alkali) photo 1 and the ones which were printed with $(25 \mathrm{~g} / \mathrm{kg})$ photo 2 . It is clear that photo n. 2 shows increasing in fabric crimping effect as a result of increasing sodium hydroxide concentration in the printing paste which affected cotton yarns and penetrate into cellulose molecules undergoes a reduction in the amount of crystallinity causing a general swelling and shrinking. Applying $\mathrm{NaOH}$ with different concentrations resulted in differential shrinkage and produce typical stretch fabric, in the printed areas. On the other hand the unprinted stripes will show clearly the crimping effect, as a result of the shrinkage effect of the printed areas, which helps in different fashion looks. ${ }^{(7,8)}$ Different concentrations of alkali resulted in different effects of crimping. .Photos can also show the differential colour tones which were achieved from dyeing of the blended fabrics with disperse dye after alkali printing, as a result of increasing polyester fibers dyeability. 


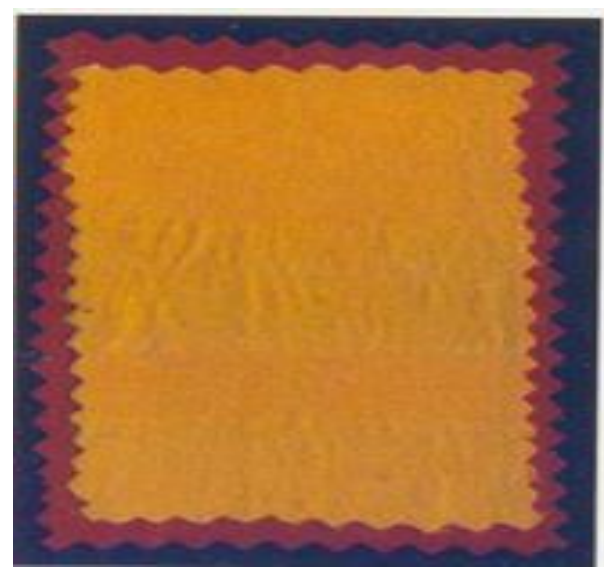

Photo (1)

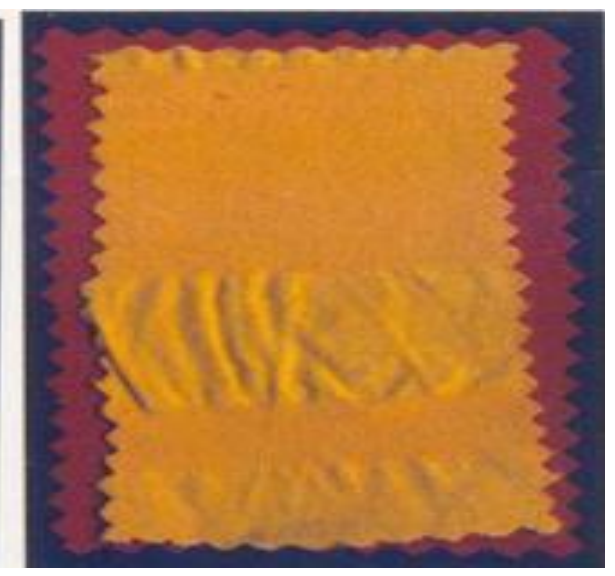

Photo (2)

Although using of sodium hydroxide on these polyester / cotton blended fabric decreased the weight of the fabric in the printed areas, it was found that in a space of $10 \mathrm{~cm}^{2}$ (including the printed and unprinted areas) the weight of the fabric increased from $1.08 \mathrm{~g}$ to $1.32 \mathrm{~g}$ as a result of crimping effect in the unprinted areas which increased the count of warp yarns and decreased the spaces between them.. On the other hand it is expected that the unprinted areas which contain the crimping effect will affect the ratio of water repellency and air permeability.

Really the repellent ratio in the unprinted stripes increased to $90 \%$, on the other hand the absorbency decreased to $10 \%$ as a result of the reduction in the spaces between the yarns which faces the water molecules. That was the opposite in the printed stripes in which the repellent ratio decreased to $10 \%$ and the absorbency increased from $50 \%$ to $90 \%$. Air permeability was also decreased as a result of space reduction between the warp yarns.

\section{Effect of steaming temperature:}

Steaming temperature plays a very important role in this experiment. After printing of the cotton / polyester blended fabrics with printing paste containing sodium hydroxide $(25 \mathrm{~g} / \mathrm{kg})$, the printed samples were exposed to different steaming temperatures $\left(105-120^{\circ} \mathrm{C}\right)$ for 20 minutes, to determine the most suitable temperature causing the desired effect. From table (2) and fig.(2-A and 2-B \}, it was obvious that increasing steaming temperature, increases the diffusion and penetration of $\mathrm{NaOH}$ inside polyester pores, assisting the alkali effect and improving colour strength from $(0.73)$ to $(0.88)$, for about $(15 \%)$ in the printed and dyed areas. When fixation temperature reaches $115^{\circ} \mathrm{C}$, it becomes a turning point at which, any 
increase in temperature will cause more weight loss which is undesired due to the decrease in tensile strength which occur, even when colour strength was increased. (9)

At high temperature, the alkali could penetrate into the cotton fibers, resulting in more even mercerization. This was probably due to the fact that, when the fibers are mercerized at a low temperature, hydrogen bonding in the crystallinity decreases. Because the bond of anhydroglucose unit group of molecular chain begins to rotate, the inaccessible hydroxyl group contained in the solution becomes accessible. Under such circumstances the number of accessible hydroxyl groups increases. However, a lower steaming temperature is better for mercerization on the surface of the fibers, rather than inside the fibers themselves. Higher steaming temperature gave better mercerization. (10) So, using steaming temperature at $115{ }^{\circ} \mathrm{C}$ was been the most suitable steaming temperature to obtain alkali effect on both polyester and cotton fibers.

Table (2) Effect of steaming temp. on working properties of polyester fabrics

\begin{tabular}{|c|c|c|c|c|c|c|}
\hline \multirow{2}{*}{ Temp ${ }^{\circ} \mathrm{C}$} & \multirow{2}{*}{$K \backslash S$} & \multirow{2}{*}{$\begin{array}{c}\text { Loss in weight } \\
(\%)\end{array}$} & \multirow{2}{*}{$\begin{array}{l}\text { Drape } \\
\text { meter }\end{array}$} & \multirow{2}{*}{$\begin{array}{c}\text { Air } \\
\text { permeability } \\
\left(\mathrm{cm}^{3} / \mathrm{cm}^{2} / \mathrm{s}\right)\end{array}$} & \multicolumn{2}{|c|}{ Water Repellency } \\
\hline & & & & & Absorption & Repellent \\
\hline 100 & 0.73 & 3.10 & 0.61 & 62.85 & $55 \%$ & $45 \%$ \\
\hline 105 & 0.74 & 6.69 & 0.50 & 64.73 & $68 \%$ & $32 \%$ \\
\hline 110 & 0.77 & 12.75 & 0.46 & 66.60 & $80 \%$ & $20 \%$ \\
\hline 115 & 0.82 & 20.0 & 0.42 & 67.9 & $90 \%$ & $10 \%$ \\
\hline 120 & 0.88 & 26.6 & 0.37 & 68.65 & $95 \%$ & $5 \%$ \\
\hline
\end{tabular}

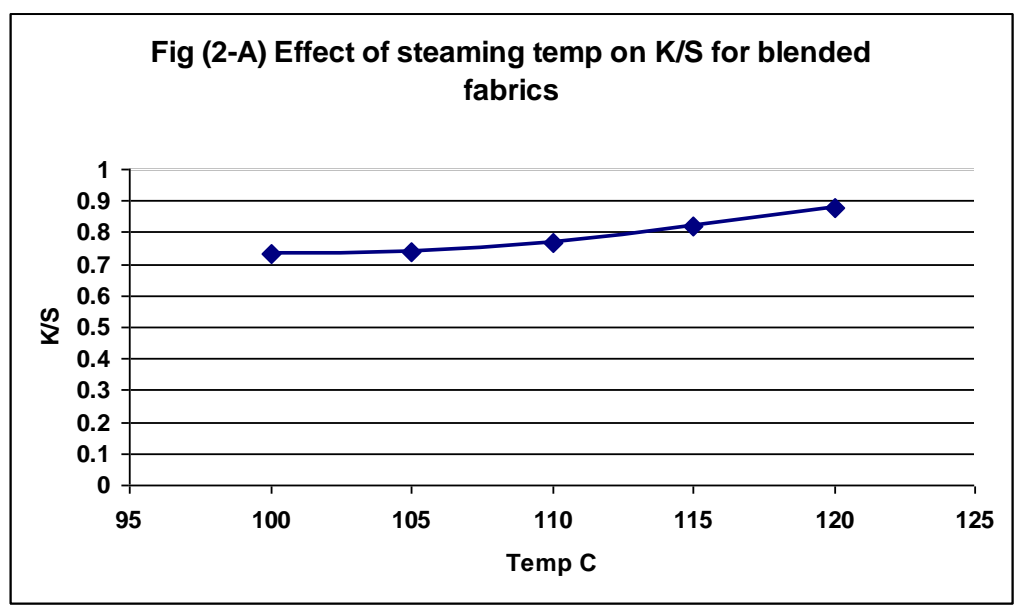




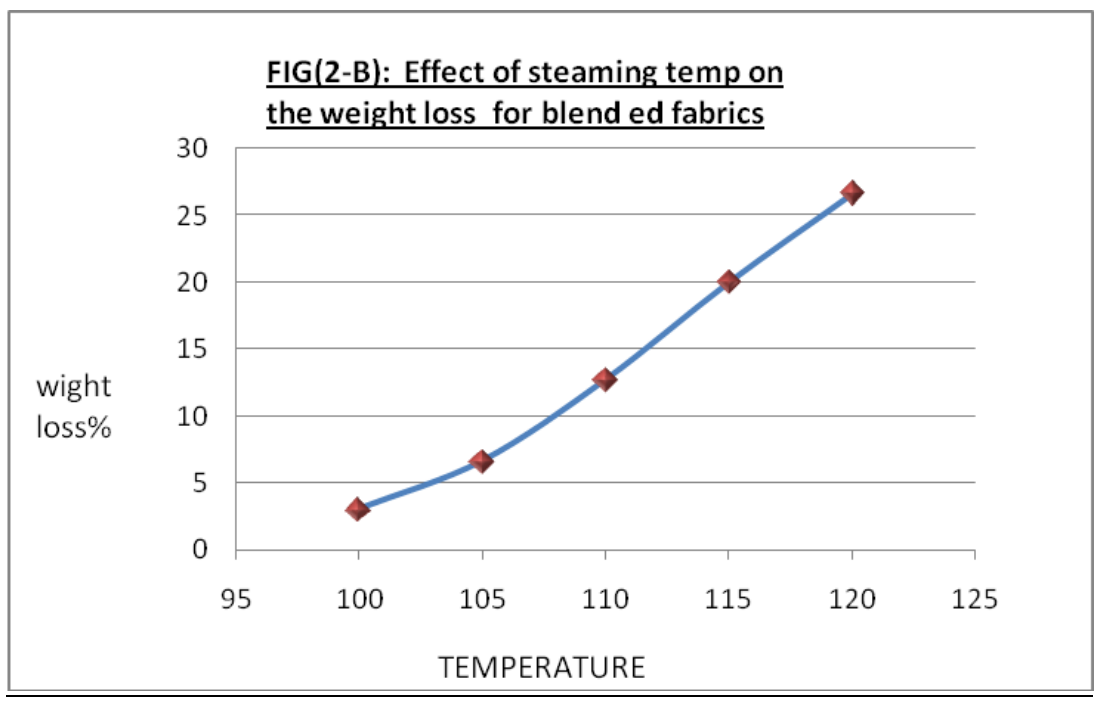

\section{Effect of steaming time:}

As in the case with most reactions the speed and extent of the reaction are strongly dependent on time and temperature. The higher the temperature and the longer reaction time, the greater in weight loss is to be expected. Steaming time is a very effective factor which affects alkali printing. The prints of blended cotton/polyester fabrics were steamed for different intervals of time, which were $(5$, $10,15,20,25 \mathrm{~min}$.) at $115^{\circ} \mathrm{C}$, using $(25 \mathrm{~g} / \mathrm{kg})$ sodium hydroxide to determine the most suitable steaming time.

From table (3) and fig. (3-A and 3-B \}, it was found that by increasing time of reaction, colour strength of dyed fabrics were increased gradually from ( 0.73 to 0.89 ) for about ( $25 \%$ ), due to the improvement of surface working properties of polyester yarns (which increased by prolonged the steaming time), specially fabric absorption . But when fixation time increased over 20 min., colour strength was decreased gradually as a result of the removal of the outer surface of polyester fibers, and the present of $\mathrm{COOH}$ groups which decrease the affinity of disperse dyes to polyester fibers. On the other hand increasing fixation time will increase weight loss and decrease tensile strength of polyester fabrics. ${ }^{(11,12)}$

Concerning cotton fibers, it was obtained, that prolonged steaming time increase cotton fibers swelling with an increasing in yellowness and tensile strength decreasing. ${ }^{(13)}$ 
Table (3) Effect of steaming time on the working properties of polyester fabrics

\begin{tabular}{|c|c|c|c|c|c|c|}
\hline \multirow{2}{*}{$\begin{array}{l}\text { Time } \\
(\min )\end{array}$} & \multirow{2}{*}{$\mathbf{K} \backslash \mathbf{S}$} & \multirow{2}{*}{$\begin{array}{c}\text { Loss in } \\
\text { weight } \\
(\%)\end{array}$} & \multirow{2}{*}{$\begin{array}{l}\text { Drape } \\
\text { meter }\end{array}$} & \multirow{2}{*}{$\begin{array}{c}\text { Air } \\
\text { permeability } \\
\left(\mathrm{cm}^{3} / \mathrm{cm}^{2} / \mathrm{s}\right)\end{array}$} & \multicolumn{2}{|c|}{ Water Repellency } \\
\hline & & & & & Absorption & Repellent \\
\hline 5 & 0.73 & 3.05 & 0.62 & 63.90 & $55 \%$ & $45 \%$ \\
\hline 10 & 0.79 & 6.65 & 0.53 & 65.00 & $69 \%$ & $31 \%$ \\
\hline 15 & 0.86 & 12.90 & 0.47 & 66.20 & $81 \%$ & $19 \%$ \\
\hline 20 & 0.89 & 20.00 & 0.42 & 67.90 & $90 \%$ & $10 \%$ \\
\hline 25 & 0.83 & 26.6 & 0.35 & 68.15 & $94 \%$ & $6 \%$ \\
\hline
\end{tabular}

Fig (3-A) Effect of time on K/S for blended fabrics
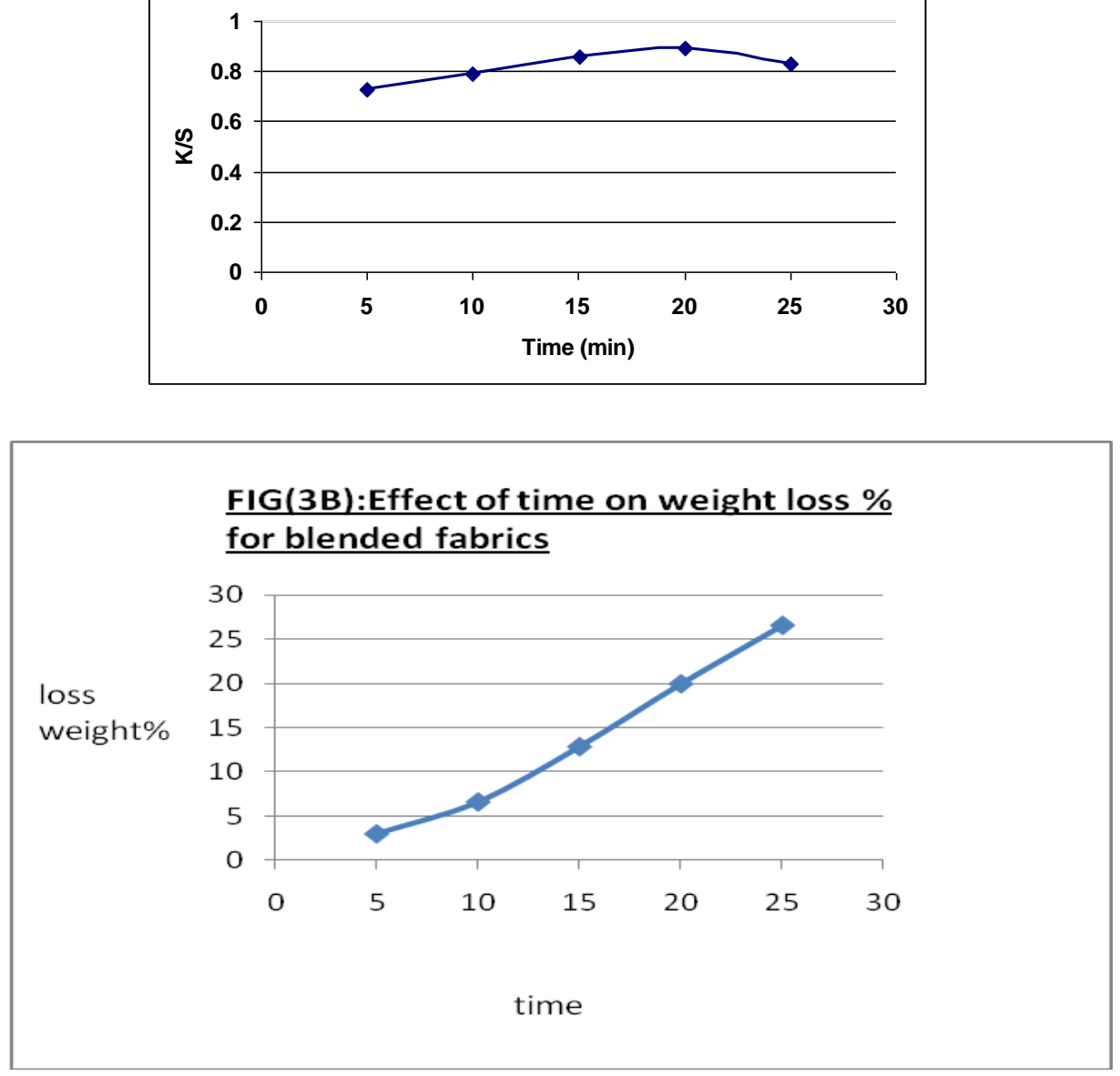


\section{Conclusion:}

50/50 cotton / polyester blended fabrics were printed with printing paste thickened with mypro gum and including $25 \mathrm{~g} / \mathrm{kg}$ sodium hydroxide, then steamed at $115{ }^{\circ} \mathrm{C}$ for 20 minutes. After steaming, the blended fabrics, were then dyed with disperse dye (foron yellow RD4 GLS GR), in order to give these blended fabrics a new fashion look, resample in the crimping effect goes to cotton yarns and the differential colour tones goes to polyester yarns. Different alkali concentrations, steaming temperature and steaming time affects the fabric physical proprieties and gave variable crimping effect.

\section{References:}

1. B.F.SMITH AND I.BLOCK, Textile in perspective, Prentice -Hall, Inc.Englewood

2. CLIFFS, N.J. P. $133-(1982)$

3. K.JENKINSON, JSDC, V. 95, P. 384 (1979)

4. V.A.SHENAI AND N.K.NAYAK, Textile dyer \& printer, n.11, P. 25-28 (1981).

5. P.G.TORTORA, Understanding Textiles, Macmillan Publishing Co. Inc. P. 319 (1978).

6. N.A.IBRAHIM , American dyestuff reporter , n. 9, P. 87- 106 (1990)

7. M.KANIT AND A. H. BAYARI, Color technology V. 119 , P.264-268 . (2003)

8. V.A.SHENAI AND N.K.NAYAK, Textile dyer \& printer, n.11, P. 25-28 (1981).

9. J.N.CHAKRABORTY,B.SUR AND S.SINGH, Colourage, n. 11 P.27-32 (2004) .

10. NOORPANAH-P,KISH-M-H, Textile-Science Journal - Vol 2 , (2001).

11. K.S.HUANG AND M.S.YEN, JSDC, V.113, n. 3 , , P. $95-98$ (1997) .

12. S.R.SHUKLA,M.R.MATHUR,V.B.HEDAOO, American Dyestuff Reporter , n.10,

13. P. 48-51 (1997).

14. P.J.HAUSER, American Dyestuff Reporter , n. 9 , P. 15-18 (1997).

15. K.S.HUANG AND M.S.YEN, JSDC, V.113, n. 3 , , P. $95-98$ (1997). 